\title{
Construction and Immunogenicity of Recombinant Vaccinia Virus Vaccine Against Japanese Encephalitis and Chikungunya Viruses Infection in Mice
}

\author{
Ying Zhang, ${ }^{1, *}$ Ji-cheng Han, ${ }^{2,3,{ }^{*}}$ Jie Jing ${ }^{1,2,{ }^{*}}$ Hao Liu, ${ }^{4}$ He Zhang, ${ }^{2}$ Zhao-hui Li, ${ }^{1}$ Ning-yi Jin, ${ }^{1,2}$ and Hui-jun Lu ${ }^{2}$
}

\begin{abstract}
Japanese encephalitis virus (JEV) is recognized as a public health risk by the World Health Organization. In Asia, each year, $\sim 70,000$ people become infected with JEV, which results in $\sim 10,000$ deaths. Chikungunya virus (CHIKV) is an RNA virus, whose infection mainly causes fever, myalgia, and skin rash. Although the mortality rate is low, it seriously affects daily life. JEV and CHIKV infect humans through mosquitoes; therefore, a recombinant vaccinia virus coexpressing JEV E and CHIKV E1 proteins was constructed to prevent their concurrent infection. In this study, after mice first immunization, booster immunization was performed at 21 days postimmunization (dpi). At $35 \mathrm{dpi}$, mice were challenged with JEV and CHIKV. Specific antibodies significantly increased in the rVTTCE1-JE-EGFP group, which were significantly $(p<0.01)$ higher than those of the control groups at 35 dpi. The plaque reduction neutralization tests (JEV) of rVTT-CE1-JE-EGFP group was 1:320 at 35 dpi. Furthermore, cytokine levels and the percentage of $\mathrm{CD}^{+}{ }^{+} \mathrm{CD} 4^{+}$and $\mathrm{CD} 3^{+} \mathrm{CD}^{+}$T-lymphocytes in the rVTT-CE1-JE-EGFP group were significantly $(p<0.01)$ higher than those in the control groups at $35 \mathrm{dpi}$. After challenge, mice body weights in rVTT-CE1-JE-EGFP group were not significantly altered, and the survival rate was $100 \%$. These results showed the rVTT-CE1-JE-EGFP group elicited significant humoral and cellular immune responses, thus indicating that the recombinant vaccine may serve as a candidate for effective prevention of CHIKV and JEV infection.
\end{abstract}

Keywords: Japanese encephalitis virus, chikungunya virus, recombinant vaccinia virus, vaccine, immune response

\section{Introduction}

$\mathbf{J}$ APANESE ENCEPHALITIS VIRUS ( JEV) is a positive-strand RNA virus of the family Flaviviridae (Vaughn and Hoke 1992, Unni et al. 2011), which is mainly transmitted by Culex tritaeniorhynchus mosquitoes. In 1935, JEV was first isolated from a human case in Japan, which was isolated and passaged in mice brain. JEV caused serious viral encephalitis in human throughout Asia with $\sim 70,000$ human cases each year and $\sim$ 10,000 fatalities (Mansfield et al. 2017). Similarly, chikungunya virus (CHIKV) is an RNA virus (Alphavirus genus, Togaviridae family) first isolated in humans in Tanzania in 1953 (Ross 1956). It is mainly transmitted by Aedes aegypti and Aedes albopictus mosquitoes. Although mortality rates are rarely associated with CHIKV ( $\sim 1$ of 1000 cases)
(Borgherini et al. 2007, Lemant et al. 2008), the high incidence rate of acute debilitating polyarthralgia $(\sim 95 \%)$ (Queyriaux et al. 2008, Sissoko et al. 2008, Weaver and Lecuit 2015) and subsequent chronic polyarthralgia (1275\%) (Brighton et al. 1983, Sissoko et al. 2009) lead to significant health and financial burdens (Yang et al. 2017).

Japanese encephalitis outbreaks were reported in South and Southeast Asia in recent years (Mansfield et al. 2017). In 2005-2006, an unprecedented chikungunya fever (Powers and Logue 2007, Schwartz and Albert 2010) occurred in the Indian Ocean, and outbreaks in India, Sri Lanka, and Southeast Asia from 2007 to 2010 (Laras et al. 2005, Kumarasamy et al. 2006, Ravi 2006, Watanaveeradej et al. 2006, Her et al. 2009, Senanayake et al. 2009) were reported. According to our epidemiological investigation, JEV and

\footnotetext{
${ }^{1}$ Key Laboratory of Zoonosis Research, Ministry of Education, College of Veterinary Medicine, College of Animal Science, Jilin University, Changchun, People's Republic of China.

${ }^{2}$ Institute of Military Veterinary, Academy of Military Sciences, Changchun, People's Republic of China

${ }^{3}$ Changchun University of Chinese Medicine, Changchun, People's Republic of China.

${ }^{4}$ School of Life Sciences and Engineering, Foshan University, Foshan, People's Republic of China.

*These authors contributed equally to this work.
} 
CHIKV were also detected in mosquitoes from Yunnan province in 2018 and 2019, China (unpublished observations). It is important to prevent Japanese encephalitis and chikungunya fever in South and Southeast Asia at the same time. Therefore, a bivalent genetic engineering vaccine is needed for JEV and CHIKV prevention (Chen et al. 2017).

JEV mainly encodes the structural proteins capsid (C), premembrane/membrane (pre-M/M), and envelope glycoprotein (E), and the nonstructural proteins NS1, NS2A, NS2B, NS3, NS4A, NS4B, and NS5 (Mukhopadhyay et al. 2005). JEV E protein is the main flavivirus surface peptide, which is involved in viral attachment, fusion, penetration, and other activities. In addition, some studies suggested that $\mathrm{E}$ protein is a candidate for JEV subunit candidate vaccine (Chavez et al. 2010). $\mathrm{CHIKV}$ has two major envelope proteins, E1 and E2, which are components of the viral spikes, which facilitate viral attachment to cell surfaces and entry to the cells. The expressed E1 and E2 proteins both induce specific antibodies, and E1 was more immunogenic. However, in immunofluorescence assays in infected cells, E2 antibody showed significantly more specificity in detecting CHIKV (Abraham and Eswaran 2015).

Poxvirus is a large double-stranded DNA virus that replicates in cytoplasm (Zhang et al. 2013). Despite the global eradication of smallpox in 1979, vaccinia virus (VACV) has been used as a vaccine vector for decades. In addition, VACV TianTan (VTT) strain was isolated from skin lesions of a Chinese patient with smallpox in 1926 by Qi Changqing (Qin et al. 2013). Furthermore, VACV was used as a vector for the development of human immunodeficiency virus (HIV) vaccines (Liu et al. 2012). Previous reports showed E3L deletion mutations in Copenhagen and NYCBH VACV strains were highly attenuated and were more suitable for human vaccine vector use (Li et al. 2016, Vijaysri et al. 2008).

The aim of this study was to develop a vaccine that effectively prevents JEV and CHIKV infection. We used the E3L gene deletion mutant VACV strain TianTan as a vector to construct the recombinant vaccinia virus rVTT-JE-CE1 vaccine, which coexpresses E protein of JEV and E1 protein of CHIKV, respectively. In vivo immunogenicity of recombinant vaccine was tested in mice.

\section{Materials and Methods}

\section{Experimental animals, viruses, cells, and plasmids}

Six-week-old female BALB/c mice (Experimental Animal Center, Academy of Military Medical Science of PLA, Beijing) were used. The CHIKV virus (GenBank: KU561451.1) was provided by Yunnan Institute of Parasitic Diseases, whereas the JEV virus (GenBank: JQ086762.1) was isolated in our laboratory. The TE3L deletion mutant of recombinant VACV strain TianTan (rVTT) (Wang et al. 2012) and the shuttle vector pSTKE-EGFP plasmid (Du et al. 2012) were previously constructed. BHK-21 cells (Baby Hamster Syrian Kidney cells) were cultured in Dulbecco's modified Eagle's medium (DMEM) with 5\% fetal bovine serum (FBS) and 1\% penicillin-streptomycin at $37^{\circ} \mathrm{C}$ in a $\mathrm{CO}_{2}$ incubator.

\section{Construction of shuttle plasmids and recombinant $V A C V$}

There were three expression cassettes MCS1, MCS2, and MCS3 in the pSTKE-EGFP, containing EGFP gene in MCS3.
CHIKV E1 (GenBank: KU561451.1) and JEV whole E gene (GenBank: AF495589.1) were cloned, and respectively inserted into pSTKE-EGFP MCS1 and MCS2 to construct shuttle plasmid pSTKE-CE1-JE-EGFP (Fig. 1A).

The recombinant vaccinia virus rVTT-CE1-JE-EGFP was obtained by homologous recombination. BHK cells were infected with rVTT-EGFP at a multiplicity of infection (MOI) of 0.1 for 2-3 h; next, pSTKE-CE1-JE-EGFP plasmids were used to transfect BHK cells using Lipofectamine 3000 (Invitrogen). After 4-6 h, culture medium was aspirated and fresh medium added. Screening for fluorescent viral plaques was performed under a fluorescence microscope, as previously described (Du et al. 2012). After 15 rounds of plaque purification, rVTT-CE1-JE-EGFP was obtained.

\section{PCR analysis of the recombinant virus}

BHK cells were infected with rVTT-CE1-JE-EGFP at an MOI of 0.1 and harvested $48-60 \mathrm{~h}$ postinfection. RNA was extracted from recombinant viruses and subjected to reverse transcription to cDNA for use as PCR templates. Amplification of CHIKV E1 and JEV E gene sequences was performed in 30 cycles at $95^{\circ} \mathrm{C}$ for $30 \mathrm{~s}, 56^{\circ} \mathrm{C}$ for $30 \mathrm{~s}$, and $72^{\circ} \mathrm{C}$ for $90 \mathrm{~s}$, and a final extension at $72^{\circ} \mathrm{C}$ for $10 \mathrm{~min}$ with Ex-Taq (TAKARA) polymerase. $\mathrm{T}_{\mathrm{m}}$ values of primer sequences are given in Table 1.

\section{Western blotting analysis of recombinant viruses}

Western blot analyses of proteins collected from BHK cells infected with rVTT-CE1-JE-EGFP at an MOI of 0.1 and harvested $36-48 \mathrm{~h}$ postinfection were performed. Reactions were developed with rabbit anti-CHIKV or anti-JEV polyclonal antibodies at a 1:1000 dilution as the primary antibody and horseradish peroxidase (HRP)-labeled goat anti-rabbit immunoglobulin G (IgG; Sigma-Aldrich, St. Louis, MO) at a 1:3000 dilution as the secondary antibody.

\section{Animal immunogenicity test}

Six-week-old BALB/c mice were randomly divided in 7 groups of 12 animals. Mice in groups 1 and 2 received intramuscular (IM) administration of $2 \mathrm{~mL}$ of $1 \times 10^{7} \mathrm{TCID}_{50} / \mathrm{mL}$ rVTT-CE1-JE-EGFP, whereas mice in groups 3 and 4 were vaccinated IM with an identical dose of rVTT-EGFP, mice in groups 5 and 6 were IM injected with $2 \mathrm{~mL}$ of phosphatebuffered saline (PBS), and mice in group 7 were IM vaccinated with SA14-14-2, according to the manufacturer's instructions. Mice were boosted with the same dose at 3-week intervals after priming. Immunized mice were weekly bled from the retro-orbital sinus/plexus after priming vaccination.

\section{Enzyme-linked immunosorbent assay}

Serum samples (1:20 dilution) were tested for specific JEV and CHIKV IgG antibodies by enzyme-linked immunosorbent assay (ELISA), using as coating antigen JEV $\mathrm{E}$ and CHIKV E1 proteins in BL21 (DE3) and HRP-labeled rabbit anti-mouse IgG antibody (diluted 1:1000) for antibody detection at $450 \mathrm{~nm}$, as previously reported (Liu et al. 2017).

\section{Plaque reduction neutralization test}

JEV neutralizing antibody titer was measured as previously reported (Tsai and $\mathrm{Wu} 2015$ ). Potency was determined by 
A

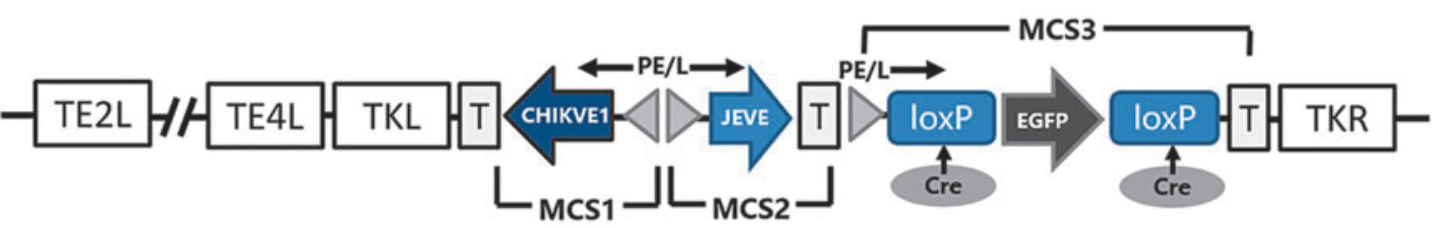

B

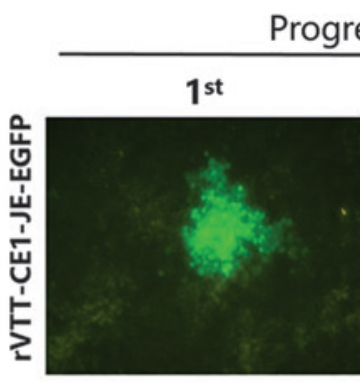

Progress screening of recombinants
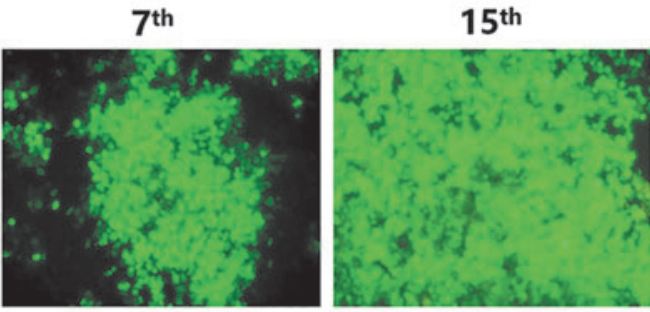

E

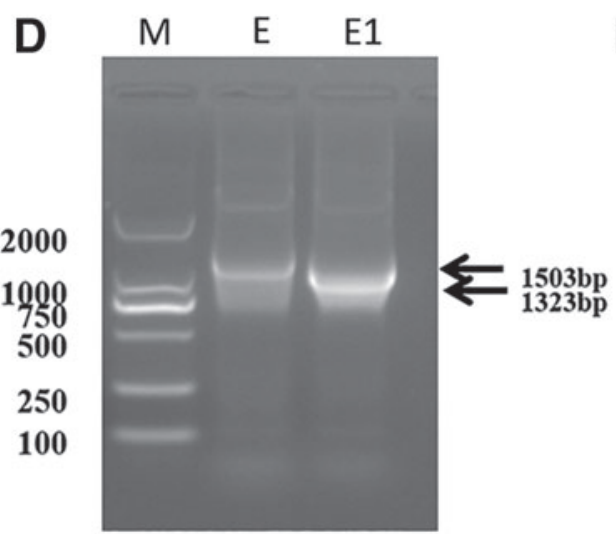

JEV- E

CHIKV-E1
C

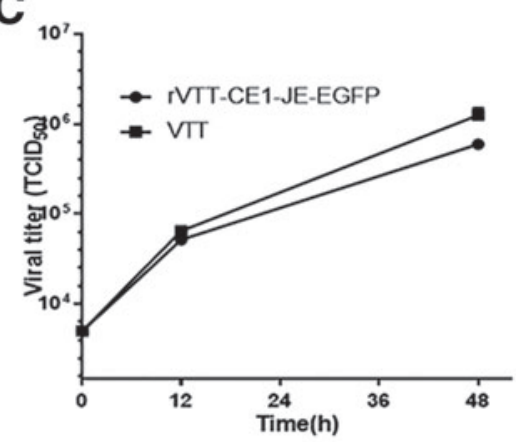

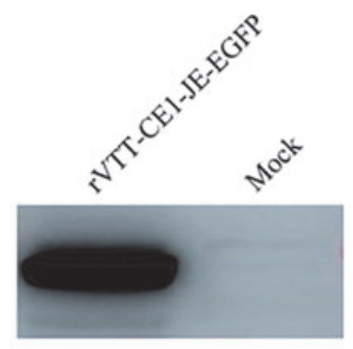

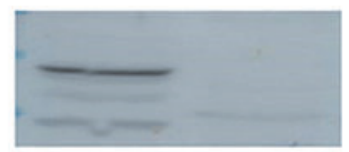

FIG. 1. Construction and identification of the recombinant vaccinia virus. (A) CHIKV-E1 and JEV-E were, respectively, inserted into MCS1 and MCS2 of pSTKE-EGFP to generate pSTKE-CE1-JE-EGFP. (B) After 15 rounds of fluorescent plaque purification, rVTT-CE1-JE-EGFP was obtained. (C) rVTT-CE1-JE-EGFP and VTT virus titers. (D) Identification of the recombinant vaccinia virus rVTT-CE1-JE-EGFP by PCR. (E) Identification of the protein in BHK cells infected with rVTT-CE1-JE-EGFP by Western blot analysis. JEV, Japanese encephalitis virus; CHIKV, chikungunya virus.

estimating the JEV neutralizing antibody response with the plaque reduction neutralization test (PRNT) in BHK cells. Twofold serial dilutions of serum samples ranged from 1:10 to 1:320. CHIKV PRNT was performed using the method of Russell et al., with modifications as previously described (Chusri et al. 2014, Russell et al. 1967). Twofold serial dilutions of serum samples started at 1:2. PRNT data were expressed as the reciprocal of the serum dilution causing $50 \%$ of plaque reduction.

\section{Cytokine analysis}

Serum samples interleukin 2 (IL-2), IL-4, and interferon $\gamma$ (IFN- $\gamma$ ) were determined by ELISA (eBioscience, San Diego,
CA) at 14 and 35 days postimmunization (dpi), according to manufacturer's instructions.

\section{Lymphocyte proliferation assay}

Spleen lymphocytes were isolated using the mice lymphocyte isolation solution kit (Hao Yang Biological Manufacturers, Tian Jin, China), following supplier's instructions at 35 dpi. Lymphocyte concentration was adjusted to $1 \times 10^{5}$ / well and proliferation was measured as previously reported (Ren et al. 2014). Each sample was stimulated with $50 \mu \mathrm{L}$ of inactivated CHIKV or JEV antigen $(10 \mu \mathrm{g} / \mathrm{mL})$ in triplicate.

Table 1. Sequences of Identification Primers and Corresponding $T_{m}$ Values

\begin{tabular}{llcr}
\hline Primers & \multicolumn{1}{c}{ Sequences $\left(5^{\prime}-3^{\prime}\right)$} & Fragment $^{\prime}($ bp $)$ & $T_{m}\left({ }^{\circ} \mathrm{C}\right)$ \\
\hline $\mathrm{F}_{\mathrm{JEV}-\mathrm{E}}$ & ATGTTTAATTGTCTGGGAATGGGC & 1503 & 56 \\
$\mathrm{R}_{\mathrm{JEV-E}}$ & TTAAGCATGCACATTGGTCGCTAAG & 1323 & 56 \\
$\mathrm{~F}_{\mathrm{CHIKV}-\mathrm{E} 1}$ & ATGTACGAACACGTAACAGTGATCCC & 56 \\
$\mathrm{R}_{\text {CHIKV-E2 }}$ & TTAGTGCCTGCTGAACGACACGCATAG & & 56 \\
\hline
\end{tabular}

JEV, Japanese encephalitis virus; CHIKV, chikungunya virus. 
Results were expressed as a stimulation index (SI), which is the ratio of stimulated sample/negative control sample.

\section{Analysis of $C D 4^{+}$and $C D 8^{+} T$ lymphocytes}

Murine spleen cells were isolated using mice lymphocyte isolation solution kit (Hao Yang Biological Manufacturers, Tianjin, China), according to manufacturer's instructions at 35 dpi. Percentages of $\mathrm{CD}^{+} \mathrm{CD}^{+}$and $\mathrm{CD}^{+} \mathrm{CD} 8^{+} \mathrm{T}$ lymphocytes were measured as previously reported (Ren et al. 2014). PE anti-mouse CD8, PE/Cy5 anti-mouse CD3, and FITC anti-mouse CD4 (BioLegend, San Diego, CA) antibodies were used for each sample. The numbers of $\mathrm{CD}^{+} \mathrm{CD}^{+}$and $\mathrm{CD}^{+} \mathrm{CD}^{+}$-positive cells in 10,000 cells were detected by flow cytometry.

\section{CHIKV and JEV challenge in mice}

At 35 dpi, mice from groups 1, 3, 5, and 7 were challenged with $1 \times 10^{4} \mathrm{TCID}_{50}$ of JEV. The other groups were challenged with $1 \times 10^{4} \mathrm{TCID}_{50}$ of CHIKV. Mice status and weight were daily monitored. After the experiment, all mice were killed.

\section{Statistical analysis}

Statistical analysis and comparisons between immunization groups were performed using the GraphPad Prism software 5.0 (San Diego, CA). The levels of cytokines, SI values, and $\mathrm{T}$ cell percentage in different groups were determined by applying one-way repeated-measures analysis of variance. $p$ value was used to describe significant statistical differences. Specifically, $p<0.01$, highly significant; $p<0.05$, significant; $p>0.05$, not significant. Data are presented as the mean \pm standard deviation (SD).

\section{Results}

\section{Confirmation of the presence of CHIKV E1 and JEV E} genes by PCR amplification

rVTT-CE1-JE-EGFP was obtained after 15 rounds of plaque purification (Fig. 1B). BHK cells were infected with recombinant VACV for $48 \mathrm{~h}$, and the virus titer was $6 \times 10^{5}$ $\mathrm{TCID}_{50} / 100 \mu \mathrm{L}$ (Fig. 1C). To confirm the presence of CHIKV E1 and JEV E genes in rVTT-CE1-JE-EGFP, PCR amplification was carried out using the primers shown in Table 1 . A $1323 \mathrm{bp}$ fragment was amplified and was consistent with CHIKV E1 gene size, whereas a 1503 bp fragment was amplified to coincide with the JEV E gene. Results showed that CHIKV E1 and JEV E genes were correctly inserted into rVTT-CE1-JE-EGFP (Fig. 1D).

\section{Analysis of rVTT-CE1-JE-EGFP expression by western blotting}

Proteins were obtained from BHK cells infected with rVTT-CE1-JE-EGFP at $0.1 \mathrm{MOI}$ and harvested $48 \mathrm{~h}$ postinfection. Western blot results showed one band of $\sim 60 \mathrm{kDa}$ consistent with JEV E protein molecular weight. The other western blot result showed one band of $\sim 50 \mathrm{kDa}$ consistent with CHIKV E1 protein molecular weight. These results indicated that CHIKV and JEV E proteins were properly expressed in BHK cells (Fig. 1E).

\section{Specific antibody responses}

CHIKV and JEV-specific antibodies by the rVTT-CE1-JEEGFP were assessed by ELISA at 0, 7, 14, 21, 28, and 35 dpi. Mice in the rVTT-CE1-JE-EGFP group exhibited JEV and CHIKV-specific antibody levels at $7 \mathrm{dpi}$. CHIKV-specific antibody titers were significantly increased at $21 \mathrm{dpi}$ in mice of the rVTT-CE1-JE-EGFP group, which further increased after boost immunization (21 dpi). At $35 \mathrm{dpi}$, CHIKVspecific antibody titers in mice of the rVTT-CE1-JE-EGFP group were significantly higher than those of the rVTT-EGFP and PBS control groups $(p<0.01)$ (Fig. 2A).

Similarly, JEV-specific antibody titers further increased after immunization. At $35 \mathrm{dpi}$, JEV-specific antibody titers in mice of the rVTT-CE1-JE-EGFP group were significantly higher than those of the rVTT-EGFP and PBS control groups $(p<0.01)$. Specific antibodies for JEV in rVTT-CE1-JEEGFP were not significantly higher than those of the SA1414-2 group at 35 dpi (Fig. 2B).

\section{Neutralizing antibody responses (PRNT)}

At 35 dpi, JEV PRNTs for mice in both rVTT-CE1-JEEGFP and SA14-14-2 groups showed high neutralizing antibodies (1:267 and 1:320), significantly higher than those of mice in the rVTT-EGFP and PBS control groups (Table 2). In addition, CHIKV PRNTs for mice in the rVTT-CE1-JEEGFP group also showed high neutralizing antibodies (1:64),
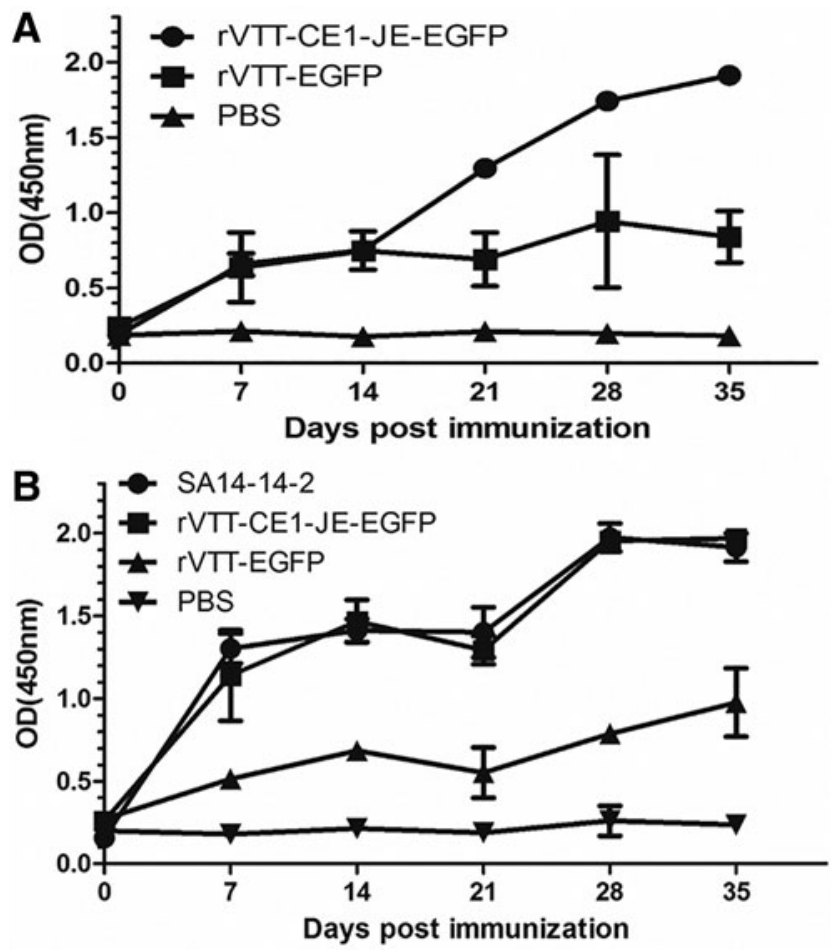

FIG. 2. Quantifying the serum antigen-specific $\operatorname{IgG}$ titer in mice after immunization. CHIKV-E1 (A) and JEV-E (B) specific antibody titers after immunizations, as measured by ELISA. Serum samples were collected from each group at 0 , $7,14,21,28$, and 35 days after vaccination. Data represent the mean \pm SD of each group. ELISA, enzyme-linked immunosorbent assay. 
Table 2. Neutralizing Antibodies for Japanese Encephalitis Virus or Chikungunya VIRUS INDICATED DAY POSTIMMUNIZATION

\begin{tabular}{|c|c|c|c|c|c|c|}
\hline \multirow[b]{3}{*}{ Vaccination groups } & \multicolumn{6}{|c|}{ Neutralizing antibody indicated day postimmunization } \\
\hline & \multicolumn{3}{|c|}{ PRNTs of JEV } & \multicolumn{3}{|c|}{ PRNTS of CHIKV } \\
\hline & 0 & 21 & 35 & 0 & 21 & 35 \\
\hline SA14-14-2 & $<10$ & $80 \pm 0$ & $320 \pm 0$ & / & I & I \\
\hline rVTT-CE1-JE-EGFP & $<10$ & $53 \pm 19$ & $267+75$ & $<2$ & $13 \pm 4$ & $64 \pm 0$ \\
\hline rVTT-EGFP & $<10$ & $<10$ & $<10$ & $<2$ & $<2$ & $<2$ \\
\hline PBS & $<10$ & $<10$ & $<10$ & $<2$ & $<2$ & $<2$ \\
\hline
\end{tabular}

PRNT, plaque reduction neutralization test.

significantly $(p<0.05)$ higher than those of mice in the VTTEGFP and PBS control groups (Table 2).

\section{Cytokine levels in immunized mice}

IL-2, IL-4, and IFN- $\gamma$ were determined to assess cytokine levels change in vaccinated mouse sera at $35 \mathrm{dpi}$. IFN- $\gamma$ (Fig. 3A) and IL-4 levels (Fig. 3B) in the rVTT-CE1-JEEGFP group were significantly $(p<0.01)$ higher than those of the rVTT-EGFP and PBS control groups at $35 \mathrm{dpi}$. Moreover, IL-2 levels (Fig. 3C) in the rVTT-CE1-JE-EGFP group were significantly $(p<0.01)$ higher than those of the rVTT-EGFP and PBS control groups at 35 dpi. At $35 \mathrm{dpi}$, IFN- $\gamma$, IL-4, and IL-2 levels in the rVTT-CE1-JE-EGFP group were, respectively, 1.14, 1.20, and 1.21 times not significantly higher than those in the SA14-14-2 group.

\section{T lymphoproliferation}

Murine spleen lymphocytes were isolated to determine T lymphocyte proliferation levels at $35 \mathrm{dpi}$. Results showed significantly $(p<0.01)$ higher $\mathrm{T}$ lymphocyte proliferation levels in the rVTT-CE1-JE-EGFP group following stimulation by inactivated $\mathrm{CHIKV}$ antigen, as compared with those of the rVTT-EGFP and PBS control groups (Fig. 4A). $\mathrm{T}$ lymphocyte proliferation levels of the rVTT-CE1-JEEGFP group after stimulation with inactivated JEV antigen were significantly $(p<0.01)$ higher than those of rVTTEGFP and PBS control groups, whereas no significant difference was observed as compared with the SA14-14-2 group at 35 dpi (Fig. 4B).

\section{$C D 4^{+}$and $C D 8^{+} T$ lymphocytes responses}

Results of $\mathrm{T}$ lymphocytes responses detected by flow cytometry showed that the percentage of $\mathrm{CD}^{+} \mathrm{CD}^{+}{ }^{+}$(Fig. 4C) and $\mathrm{CD}^{+} \mathrm{CD}^{+}$(Fig. 4D) $\mathrm{T}$ lymphocytes in the rVTT-CE1JE-EGFP group were significantly $(p<0.01)$ higher than those of the PBS control group at $35 \mathrm{dpi}$. $\mathrm{CD}^{+} \mathrm{CD}^{+}$and $\mathrm{CD}^{+} \mathrm{CD}^{+} \mathrm{T}$ lymphocytes percentage in the rVTT-CE1-JEEGFP group was not significantly higher than that in the rVTT-EGFP control group at 35 dpi.

\section{Body weight change and survival after challenge}

To assess the protective effect of rVTT-CE1-JE-EGFP against JEV and CHIKV infection, the status and body weight of mice was daily monitored. Weight of mice challenged with JEV (Fig. 5A) or CHIKV (Fig. 5B) in the rVTT-
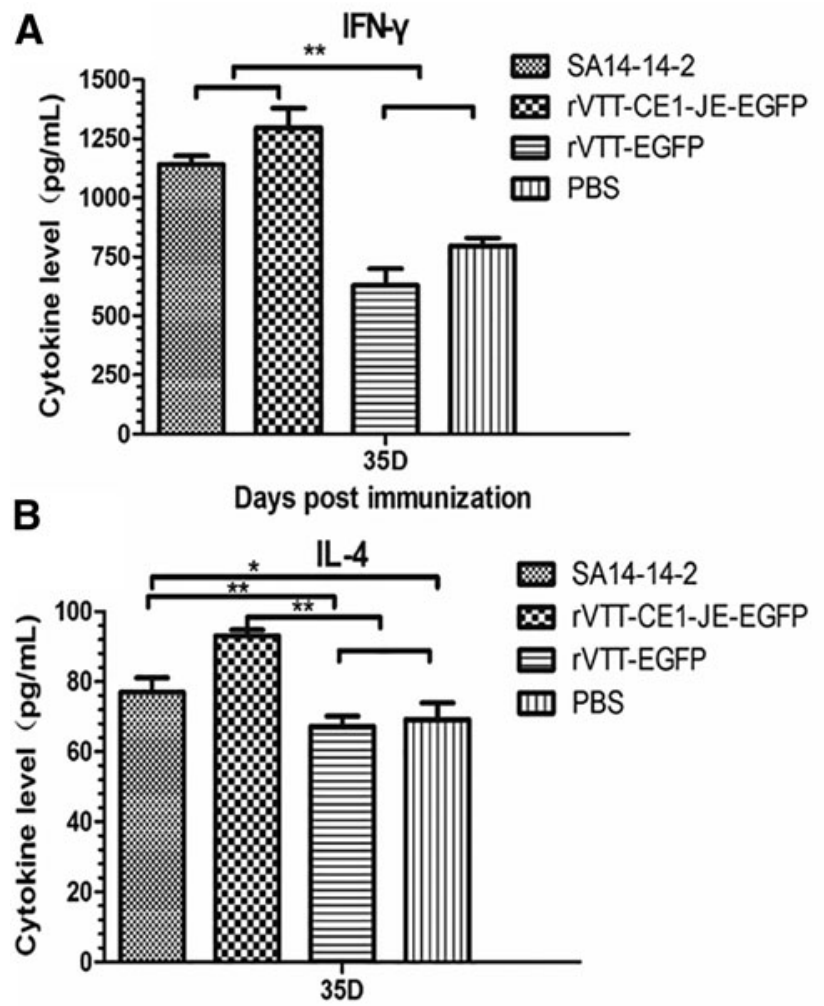

Days post immunization

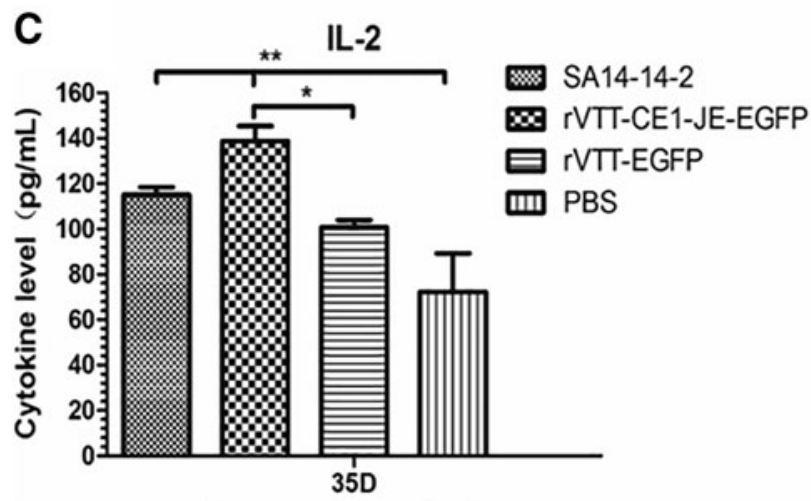

Days post immunization

FIG. 3. Serum cytokine levels. IFN- $\gamma$ (A), IL-4 (B), and IL2 (C) levels from each group. Data represent the mean \pm SD. ${ }^{*} p<0.05 ; * * p<0.01$. IFN- $\gamma$, interferon $\gamma$; IL, interleukin. 

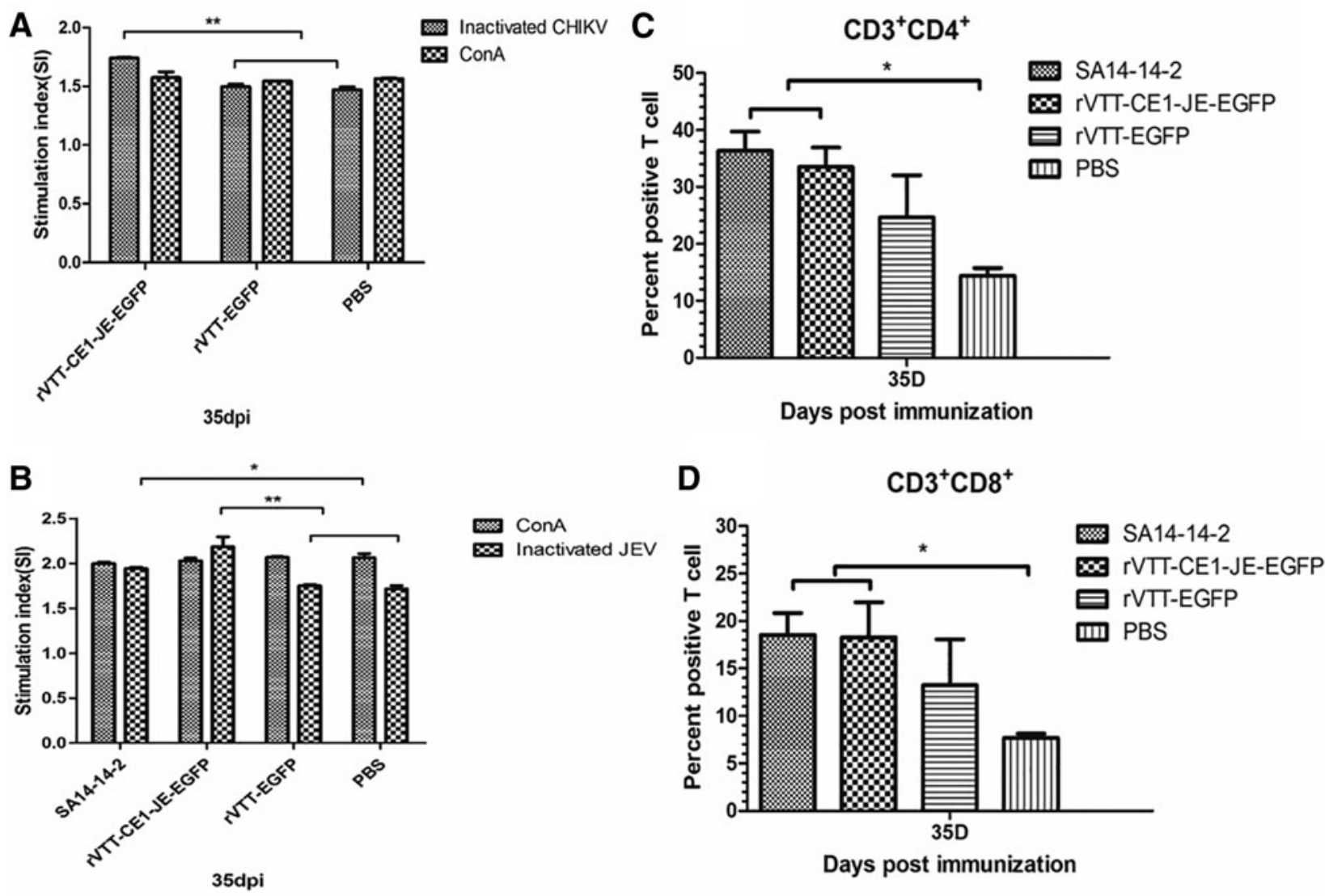

FIG. 4. T lymphocyte proliferation and $\mathrm{CD}^{+} \mathrm{CD}^{+}$and $\mathrm{CD} 3^{+} \mathrm{CD} 8^{+} \mathrm{T}$ lymphocytes percentage in each group. Analysis of $\mathrm{T}$ lymphocyte proliferative responses in mice immunized with rVTT-CE1-JE-EGFP, rVTT-EGFP or PBS, and stimulated with inactivated CHIKV (A) or JEV (B) antigen epitope peptides, percentages of $\mathrm{CD}^{+} \mathrm{CD}^{+}(\mathbf{C})$ and $\mathrm{CD} 3^{+} \mathrm{CD} 8^{+}$, and $(\mathbf{D}) \mathrm{T}$ lymphocytes from each group immunized with rVTT-CE1-JE-EGFP, rVTT-EGFP or PBS. ConA is a positive control in lymphocyte proliferation assay. SI indicates proliferation potential of $\mathrm{T}$ lymphocytes from each group. Data represent mean \pm SD. $* p<0.05 ; * * p<0.01$.

CE1-JE-EGFP group was not altered. However, mice challenged with JEV or CHIKV in the rVTT-EGFP and PBS groups continuously lost weight. After JEV challenge, survival rate in the rVTT-CE1-JE-EGFP, SA14-14-2, rVTTEGFP, and PBS groups were $100 \%, 100 \%, 33.3 \%$, and $0 \%$ at 10 days postchallenge (dpc), respectively (Fig. 5C). Moreover, survival rate of mice challenged with CHIKV in the rVTT-CE1-JE-EGFP, rVTT-EGFP, and PBS groups was $100 \%, 80 \%$, and $50 \%$ respectively, at $10 \mathrm{dpc}$ (Fig. 5D). Results showed that rVTT-CE1-JE-EGFP had a protective effect after JEV and CHIKV challenge.

\section{Discussion}

JEV is a zoonotic disease transmitted to human and swine by mosquitoes (Mansfield et al. 2017, Nickols et al. 2017). WHO 2015 report showed that JEV has spread to humans in 24 countries in the Western Pacific and Southeast Asia (Nickols et al. 2017). There are currently four types of vaccines for JEV, including inactivated mouse brain-derived JEV, inactivated Vero cells-produced JEV, live-attenuated JEV, and live-recombinant vaccines (Nickols et al. 2017). At present, SA14-14-2 live-attenuated vaccine developed by China is one of the most widely used vaccines.
In this study, we constructed a recombinant VACV that expresses JEV E and CHIKV E1 proteins with the VACV TianTan E3L deletion mutant, rVTT-CE1-JE-EGFP. With regard to JEV vaccine, specific antibodies significantly increased, as previously reported (Lee et al. 2016). At $35 \mathrm{dpi}$, JEV-specific antibodies in the rVTT-CE1-JE-EGFP group were significantly higher than those in the rVTT-EGFP and PBS control groups $(p<0.01)$, but there was no significant difference as compared with the SA14-14-2 group. At $35 \mathrm{dpi}$, rVTT-CE1-JE-EGFP PRNT was 1:267, and there was no significant difference compared with that of the SA14-14-2 group. Previous studies reported that immunized mice produced PRNTs similar to the results of this study (Nam et al. 1999), and suggested that PRNTs (1:160) are sufficient to completely protect mice against a JEV challenge (Lee et al. 2016). Sera cytokine levels and $\mathrm{CD}^{+} \mathrm{CD}^{+}$and $\mathrm{CD}^{+}{ }^{+} \mathrm{CD} 8^{+}$ T lymphocytes percentage in the rVTT-CE1-JE-EGFP group were significantly higher than those in the rVTT-EGFP $(p<0.01)$ and PBS $(p<0.01)$ control groups, but there was no significant difference compared with those of the SA1414-2 group, thus indicating that rVTT-CE1-JE-EGFP effectively induced humoral and cellular responses. After murine JEV challenge, body weights in the rVTT-CE1-JE-EGFP group was not significantly altered, and the survival rate was 

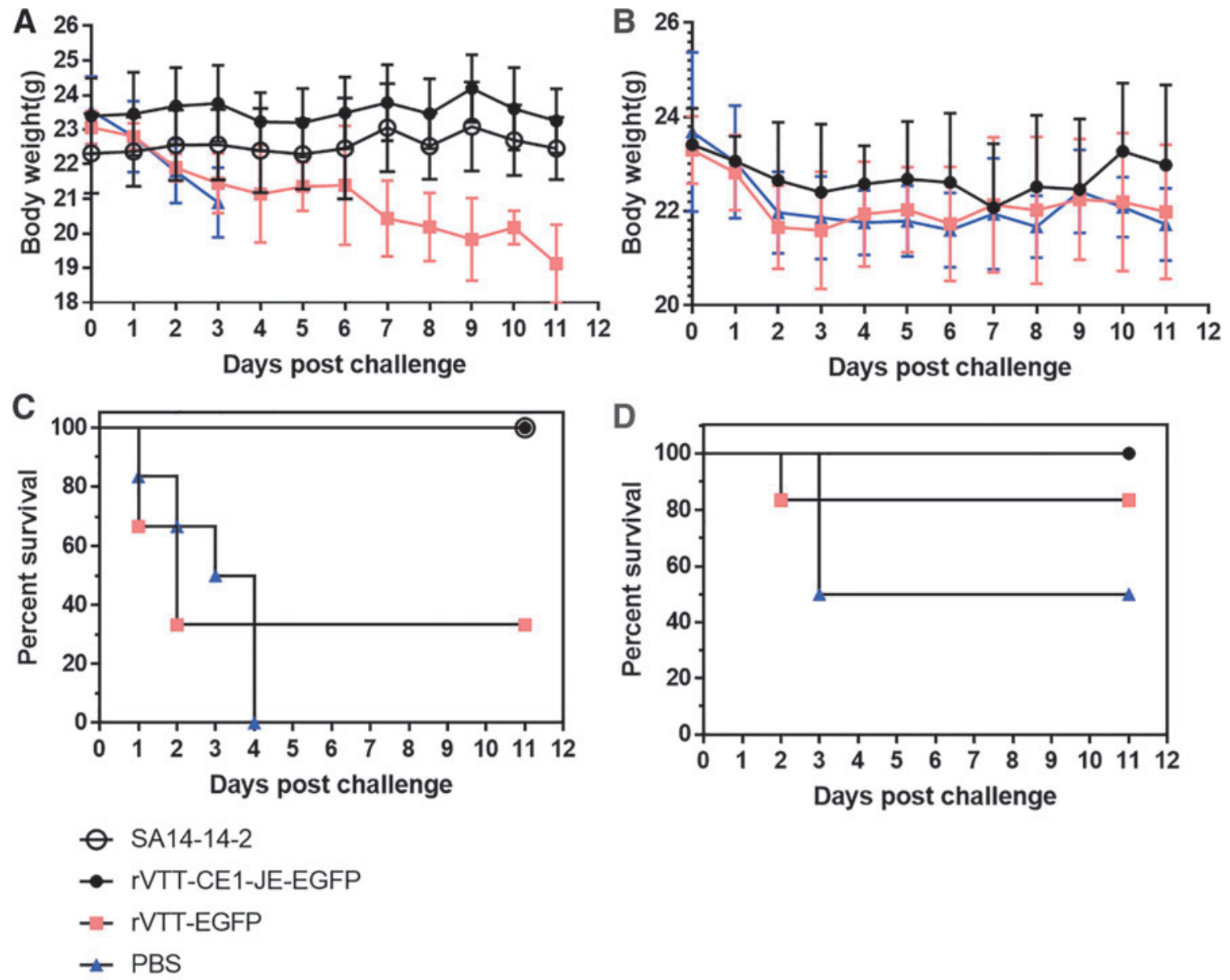

FIG. 5. Vaccine protective effect. (A) Body weight change of each group after JEV challenge. (B) Survival rate of mice in each group after JEV challenge. (C) Body weight change of each group after CHIKV challenge. (D) Survival rate of mice in each group after CHIKV challenge.

$100 \%$. These results indicate that rVTT-CE1-JE-EGFP was protective against JEV in mice.

At 35 dpi, CHIKV-specific antibodies in the rVTT-CE1JE-EGFP group were significantly higher than those of the control group $(p<0.01)$, suggesting that mice produce E1specific antibodies to provide a protective effect against CHIKV infection. Previous studies have shown recombinant MVA expressing the structural envelope of CHIKV-induced levels of neutralizing antibodies ranging from 40 to 160 at 63 dpi. Animals immunized with recombinant MVA vaccine and challenged with CHIKS27 had null or marginal levels of viral RNA detectable in different tissues (van den Doel et al. 2014). In our study, CHIKV PRNTs in the rVTT-CE1-JEEGFP group was 1:64 at $35 \mathrm{dpi}$, which was similar to the recombinant MVA vaccine study.

Some studies have shown that $\mathrm{T}$ cells are important effector cells in viral infection. $\mathrm{CD}^{+}$and $\mathrm{CD}^{+}$play an important role in virus infection in mice ( $\mathrm{Ng} 2017)$. To date, reports on the role of $\mathrm{T}$ cells in CHIKV patients are limited, indicating that $\mathrm{CD}^{+}$is predominantly present in the early stages of the disease and that $\mathrm{CD}^{+}$appears at later stages of the disease to help produce CHIKV-specific humoral immunity (Wauquier et al. 2011). Some studies have shown that CD4 plays a critical role in joint inflammation (Teo et al.
2015, Teo et al. 2013). In our study, $\mathrm{CD}^{+} \mathrm{CD}^{+}$and $\mathrm{CD}^{+}{ }^{+} \mathrm{CD} 8^{+}$percentage in the rVTT-CE1-JE-EGFP group was significantly higher than that in the control group $(p<0.05)$ at $35 \mathrm{dpi}$. At $35 \mathrm{dpi}$, CHIKV neutralizing antibody titer was 1:64, which was significantly higher than that of the control group. Neutralizing antibody titers were similar that those of previous studies (Eldi et al. 2017). After CHIKV challenge in mice, weight of mice in rVTT-CE1-JE-EGFP group was not significantly altered, which indicated rVTTCE1-JE-EGFP induced partial protection. Because BALB/c mice is not very susceptible to CHIKV (5/10 died in PBS group), immunodeficient AG129 mice will be chosen for future CHIKV challenge, which lack type I and II interferon receptor.

\section{Conclusions}

JEV and CHIKV may concurrently infect humans by mosquitoes, thus recombinant VACV coexpressing the JEV $\mathrm{E}$ and CHIKV E1 was constructed for JEV and CHIKV prevention. These results suggest that recombinant VACV induced humoral and cellular immune responses in mice, and rVTT-CE1-JE-EGFP prevented JEV and CHIKV infection in mice. 


\section{Statement of Ethics}

All animal protocols were approved by the Animal Care and Use Committee of the Institute of Military Veterinary. Mice used in the experiment were humanely bred and killed at the end of the study.

\section{Acknowledgment}

The authors thank the native English-speaking scientists of EditSprings for editing our article.

\section{Author Disclosure Statement}

The research was conducted in the absence of any commercial or financial relationships that could be construed as a potential conflict of interest.

\section{Funding Information}

This work was supported by the National Program on Key Research Project of China (no. 2016YFD0500401), and National Science and Technology Major Project (no. 2018ZX10101003 and no. 2018ZX10102).

\section{References}

Abraham R, Eswaran S. Comparison of Chikungunya virus E1 and E2 envelope proteins and their antibodies with respect to immunogencity and efficiency in virus detection. Allapuzha, India: 27th Kerala Science Congress, 2015.

Borgherini G, Poubeau P, Staikowsky F, Lory M, et al. Outbreak of chikungunya on Reunion Island: Early clinical and laboratory features in 157 adult patients. Clin Infect Dis 2007; 44:1401-1407.

Brighton SW, Prozesky OW, de la Harpe AL. Chikungunya virus infection. A retrospective study of 107 cases. S Afr Med J 1983; 63:313-315.

Chavez JH, Silva JR, Amarilla AA, Moraes Figueiredo LT. Domain III peptides from flavivirus envelope protein are useful antigens for serologic diagnosis and targets for immunization. Biologicals 2010; 38:613-618.

Chen TH, Hu CC, Liao JT, Lee YL, et al. Production of Japanese encephalitis virus antigens in plants using bamboo mosaic virus-based vector. Front Microbiol 2017; 8:788.

Chusri S, Siripaitoon P, Silpapojakul K, Hortiwakul T, Charernmak B, Chinnawirotpisan P, Nisalak A, Thaisomboonsuk B, Klungthong C, Gibbons RV, Jarman RG. Kinetics of chikungunya infections during an outbreak in Southern Thailand, 2008-2009. Am J Trop Med Hyg 2014; 90:410 417.

Du S, Li C, Wang Y, Liu C, et al. Construction and evaluation of a new triple-gene expression cassette vaccinia virus shuttle vector. J Virol Methods 2012; 185:175-183.

Eldi P, Cooper TH, Liu L, Prow NA, et al. Production of a chikungunya vaccine using a $\mathrm{CHO}$ cell and attenuated viralbased platform technology. Mol Ther 2017; 25:2332-2344.

Her Z, Kam YW, Lin RT, Ng LF. Chikungunya: a bending reality. Microbes Infect 2009; 11:1165-1176.

Kumarasamy V, Prathapa S, Zuridah H, Chem YK, et al. Reemergence of Chikungunya virus in Malaysia. Med J Malaysia 2006; 61:221-225.

Laras K, Sukri NC, Larasati RP, Bangs MJ, et al. Tracking the re-emergence of epidemic chikungunya virus in Indonesia. Trans R Soc Trop Med Hyg 2005; 99:128-141.
Lee EY, Kim JY, Lee DK, Yoon IS, et al. Sublingual immunization with Japanese encephalitis virus vaccine effectively induces immunity through both cellular and humoral immune responses in mice. Microbiol Immunol 2016; 60:846-853.

Lemant J, Boisson V, Winer A, Thibault L, et al. Serious acute chikungunya virus infection requiring intensive care during the Reunion Island outbreak in 2005-2006. Crit Care Med 2008; 36:2536-2541.

Li Y, Sheng Y, Chu Y, Ji H, et al. Seven major genomic deletions of vaccinia virus Tiantan strain are sufficient to decrease pathogenicity. Antiviral Res 2016; 129:1-12.

Liu L, Hao Y, Luo Z, Huang Y, et al. Broad HIV-1 neutralizing antibody response induced by heterologous gp140/gp145 DNA prime-vaccinia boost immunization. Vaccine 2012; 30: 4135-4143.

Liu X, Fang Y, Zhou P, Lu Y, et al. Chimeric virus-like particles elicit protective immunity against serotype $O$ footand-mouth disease virus in guinea pigs. Appl Microbiol Biotechnol 2017; 101:4905-4914.

Mansfield KL, Hernandez-Triana LM, Banyard AC, Fooks AR, et al. Japanese encephalitis virus infection, diagnosis and control in domestic animals. Vet Microbiol 2017; 201:85-92.

Mukhopadhyay S, Kuhn RJ, Rossmann MG. A structural perspective of the flavivirus life cycle. Nat Rev Microbiol 2005; 3:13-22.

Nam JH, Wyatt LS, Chae SL, Cho HW, et al. Protection against lethal Japanese encephalitis virus infection of mice by immunization with the highly attenuated MVA strain of vaccinia virus expressing JEV prM and E genes. Vaccine 1999; 17: 261-268.

$\mathrm{Ng}$ LFP. Immunopathology of chikungunya virus infection: Lessons learned from patients and animal models. Annu Rev Virol 2017; 4:413-427.

Nickols B, Tretyakova I, Tibbens A, Klyushnenkova E, et al. Plasmid DNA launches live-attenuated Japanese encephalitis virus and elicits virus-neutralizing antibodies in BALB/c mice. Virology 2017; 512:66-73.

Powers AM, Logue $\mathrm{CH}$. Changing patterns of chikungunya virus: Re-emergence of a zoonotic arbovirus. J Gen Virol 2007; 88(Pt 9):2363-2377.

Qin L, Liang M, Evans DH. Genomic analysis of vaccinia virus strain TianTan provides new insights into the evolution and evolutionary relationships between Orthopoxviruses. Virology 2013; 442:59-66.

Queyriaux B, Simon F, Grandadam M, Michel R, et al. Clinical burden of chikungunya virus infection. Lancet Infect Dis 2008; 8:2-3.

Ravi V. Re-emergence of chikungunya virus in India. Indian J Med Microbiol 2006; 24:83-84.

Ren JQ, Sun WC, Lu HJ, Wen SB, et al. Construction and immunogenicity of a DNA vaccine coexpressing GP3 and GP5 of genotype-I porcine reproductive and respiratory syndrome virus. BMC Vet Res 2014; 10:128.

Ross RW. The Newala epidemic. III. The virus: Isolation, pathogenic properties and relationship to the epidemic. J Hyg (Lond) 1956; 54:177-191.

Russell PK, Nisalak A, Sukhavachana P, Vivona S. A plaque reduction test for dengue virus neutralizing antibodies. J Immunol 1967; 99:285-290.

Schwartz O, Albert ML. Biology and pathogenesis of chikungunya virus. Nat Rev Microbiol 2010; 8:491-500.

Senanayake MP, Senanayake SM, Vidanage KK, Gunasena S, et al. Vertical transmission in chikungunya infection. Ceylon Med J 2009; 54:47-50. 
Sissoko D, Malvy D, Ezzedine K, Renault P, Moscetti F, Ledrans M, Pierre V. Post-epidemic Chikungunya disease on Reunion Island: Course of rheumatic manifestations and associated factors over a 15-month period. PLoS Negl Trop Dis 2009; 3:e389.

Sissoko D, Moendandze A, Malvy D, Giry C, et al. Seroprevalence and risk factors of chikungunya virus infection in Mayotte, Indian Ocean, 2005-2006: A population-based survey. PLoS One 2008; 3:e3066.

Teo TH, Her ZS, Tan JJL, Lum FM, et al. Caribbean and La Reunion Chikungunya virus isolates differ in their capacity to induce proinflammatory Th1 and NK cell responses and acute joint pathology. J Virol 2015; 89:7955-7969.

Teo TH, Lum FM, Claser C, Lulla V, et al. A Pathogenic Role for CD4(+) T Cells during chikungunya virus infection in mice. J Immunol 2013; 190:259-269.

Tsai HC, Wu R. Mechanisms of Cholera Toxin in the Modulation of TH17 Responses. Crit Rev Immunol 2015; 35:135152.

Unni SK, Ruzek D, Chhatbar C, Mishra R, et al. Japanese encephalitis virus: From genome to infectome. Microbes Infect $2011 ; 13: 312-321$.

van den Doel P, Volz A, Roose JM, Sewbalaksing VD, et al. Recombinant modified vaccinia virus Ankara expressing glycoprotein E2 of Chikungunya virus protects AG129 mice against lethal challenge. PLoS Negl Trop Dis 2014; 8:e3101.

Vaughn DW, Hoke CH, Jr. The epidemiology of Japanese encephalitis: Prospects for prevention. Epidemiol Rev 1992; 14: 197-221.

Vijaysri S, Jentarra G, Heck MC, Mercer AA, et al. Vaccinia viruses with mutations in the $\mathrm{E} 3 \mathrm{~L}$ gene as potential replication-competent, attenuated vaccines: Intra-nasal vaccination. Vaccine 2008; 26:664-676.

Wang Y, Kan S, Du S, Qi Y, et al. Characterization of an attenuated TE3L-deficient vaccinia virus Tian Tan strain. Antiviral Res 2012; 96:324-332.
Watanaveeradej V, Endy TP, Simasathien S, Kerdpanich A, et al. The study transplacental chikungunya virus antibody kinetics, Thailand. Emerg Infect Dis 2006; 12:1770-1772.

Wauquier N, Becquart P, Nkoghe D, Padilla C, et al. The acute phase of chikungunya virus infection in humans is associated with strong innate immunity and T CD8 cell activation. J Infect Dis 2011; 204:115-123.

Weaver SC, Lecuit M. Chikungunya virus and the global spread of a mosquito-borne disease. N Engl J Med 2015; 372:1231-1239.

Yang S, Fink D, Hulse A, Pratt RD. Regulatory considerations in development of vaccines to prevent disease caused by Chikungunya virus. Vaccine 2017; 35:4851-4858.

Zhang Q, Tian M, Feng Y, Zhao K, et al. Genomic sequence and virulence of clonal isolates of vaccinia virus Tiantan, the Chinese smallpox vaccine strain. PLoS One 2013; 8:e60557.

Address correspondence to: Ning-yi Jin

Key Laboratory of Zoonosis Research Ministry of Education College of Veterinary Medicine College of Animal Science Jilin University Changchun 130062 People's Republic of China

E-mail: jinningyi2000@126.com

Hui-jun Lu Institute of Military Veterinary Academy of Military Sciences Changchun 130122 People's Republic of China

E-mail: huijun_lu@126.com 